\section{Montreal Cognitive Assessment and Magnetic Resonance Imaging Identify Cognitive Impairment in Young Patients with Neuropsychiatric Systemic Lupus Erythematosus}

\section{To the Editor:}

We read with great interest the paper on performance of screening tests for cognitive impairment (CI) in systemic lupus erythematosus (SLE), by Nantes, $e t a l^{1}$. They indicated that CI is highly prevalent in SLE, and that the Montreal Cognitive Assessment (MoCA) is a good tool to screen early CI. In addition, patients with more years of education were less likely to have CI.

We agree with the authors' opinion and would like to emphasize the importance of regular MoCA and imaging examinations in patients with a history of neuropsychiatric SLE (NPSLE). The tests help identify insidious CI. We presented 2 young women with SLE who had clinical early-onset dementia and brain atrophy on magnetic resonance imaging (MRI). The clinical research has been approved by Tri-Service General Hospital, Taiwan (TSGHIRB No.: 1-106-05-109).

The first case is a 33-year-old female, educated to master's degree level, who was diagnosed with SLE 3 years before manifestations of CI. She had been admitted for SLE flare with class IV lupus nephritis, mentality changes, incoherent speech, and mild dysarthria. The blood analysis showed a high serum level of anti-dsDNA antibodies, anticardiolipin IgG, lupus anticoagulant (LAC), and antiribosomal P antibody. Her brain MRI showed cerebral atrophy compared to age-matched healthy people or SLE patients without NPSLE. The other patient, a 40-year-old female, was a vocational school graduate who experienced 10 years of class IV lupus nephritis. The laboratory study revealed low serum complement and a high level of anti-dsDNA antibodies, $\beta_{2}$-glycoprotein I, and LAC. Recurrent fluctuated confusion, emotional changes, and dyscalculia have developed for 1 year. The brain MRI revealed symmetrical cortical atrophy at frontotemporoparietal lobes, compared to age-matched healthy people or SLE patients without NPSLE.

A cross-sectional study showed that patients with SLE had a higher risk of dementia than those without SLE ${ }^{2}$. Three possible causes of dementia in SLE include recurrent cerebral microinfarction in patients with SLE caused by antiphospholipid antibodies (aPL), steroid-related impaired attention, and the neuroanatomical structural changes in patients with SLE, especially size reduction in the hippocampus, causing impaired memory. These 2 well-educated young women did not fulfill the diagnostic criteria of aPL syndrome and the corticosteroid-induced psychiatric disorder ${ }^{3}$. The most common radiographic findings in NPSLE include hyperintensities in the white or grey matter, parenchymal defect, and focal atrophy ${ }^{4}$. Brain MRI of our patients showed no evidence of microinfarction or other structural changes except cerebral atrophy. We should be more aware of the risk of CI in patients with NPSLE. Cognition function and brain image check as routine followup measures are recommended for young patients with a history of NPSLE.

YA-CHI LI, MD, Division of Rheumatology/Immunology and Allergy, Department of Internal Medicine, Tri-Service General Hospital, National Defense Medical Center; DEH-MING CHANG, PhD, Division of Rheumatology/Immunology and Allergy, Department of Internal Medicine, Tri-Service General Hospital, National Defense Medical Center, and Division of Allergy, Immunology, Rheumatology, Department of Internal Medicine, Taipei Veteran General Hospital; CHUN-CHI LU, MD, Division of Rheumatology/Immunology and Allergy, Department of Internal Medicine, Tri-Service General Hospital, National Defense Medical Center, Taipei, Taiwan. Address correspondence to Dr. C.C. Lu, No. 325, Sec 2, Cheng-Gong Rd, Neihu Dist., 114, Taipei, Taiwan, ROC. E-mail: jameslutaiwan@gmail.com

\section{REFERENCES}

1. Nantes SG, Su J, Dhaliwal A, Colosimo K, Touma Z. Performance of screening tests for cognitive impairment in systemic lupus erythematosus. J Rheumatol 2017;44:1583-9.

2. Lin YR, Chou LC, Chen HC, Liou TH, Huang SW, Lin HW. Increased risk of dementia in patients with systemic lupus erythematosus: a nationwide population-based cohort study. Arthritis Care Res 2016;68:1774-9.

3. Chau SY, Mok CC. Factors predictive of corticosteroid psychosis in patients with systemic lupus erythematosus. Neurology 2003;61:104-7.

4. Luyendijk J, Steens SC, Ouwendijk WJ, Steup-Beekman GM, Bollen EL, van der Grond J, et al. Neuropsychiatric systemic lupus erythematosus: lessons learned from magnetic resonance imaging. Arthritis Rheum 2011;63:722-32.

J Rheumatol 2019;46:6; doi:10.3899/jrheum.181231 